\title{
INTERVENÇÃO FONOAUDIOLÓGICA EM RECÉM-NASCIDO PRÉ-TERMO: ESTIMULAÇÃO OROMOTORA E SUCÇÃO NÃO-NUTRITIVA
}

\author{
Preterm Newborns speech therapy: oromotor stimulation \\ and non-nutritive sucking
}

Diane Fernanda Bernal Calado ${ }^{(1)}$, Rosana de Souza ${ }^{(2)}$

\begin{abstract}
RESUMO
Tema: intervenção fonoaudiológica em recém-nascido pré-termo, com uso das técnicas de estimulação oromotora e sucção não-nutritiva. Procedimentos: participaram da intervenção fonoaudiológica dois recém-nascidos gemelares com idade gestacional corrigida de 35 semanas e 2/7 dias. Foi realizada a avaliação fonoaudiológica para verificar a prontidão do prematuro para o início da alimentação por via oral e a eficiência da alimentação por via oral por meio das relações: volume aceito pelo volume total prescrito e volume aceito pelo tempo de aceitação da dieta. Posteriormente foi sorteada a técnica que cada recém-nascido receberia. RN1 recebeu a técnica de estimulação oromotora e o RN2 recebeu a técnica de sucção não-nutritiva. Em seguida, receberam 10 sessões de terapia fonoaudiológica e foram reavaliados. Resultados: após as sessões de estimulação os recémnascidos apresentaram melhora nos reflexos orais e no padrão de sucção não nutritiva e melhoraram no aproveitamento da dieta oral. Conclusão: ambos os recém-nascidos foram beneficiados com a intervenção fonoaudiológica.
\end{abstract}

DESCRITORES: Recém-Nascido; Lactente; Alimentação; Deglutição; Transtornos da Deglutição; Técnicas

\section{INTRODUÇÃO}

Atualmente com o uso das novas tecnologias médico hospitalares nas áreas neonatal e perinatal, os recém-nascidos pré-termo (RNPT) - abaixo de 37 semanas de idade gestacional - e de baixo peso ao nascimento - peso inferior a 2500 gramas - têm mais chances de sobrevida ${ }^{1}$. Pesquisas revelam que no final da década de 90, no Brasil, cerca de

(1) Fonoaudióloga; Aprimoranda do Programa de Aprimoramento Profissional da Secretaria de Estado da Saúde na área de Fonoaudiologia em Neonatologia do Hospital Maternidade Leonor Mendes de Barros, HMLMB, São Paulo, SP, Brasil.

(2) Fonoaudióloga; Supervisora do Programa de Aprimoramento Profissional da Secretaria de Estado da Saúde na área de Fonoaudiologia em Neonatologia do Hospital Maternidade Leonor Mendes de Barros, HMLMB, São Paulo, SP, Brasil; Mestre em Linguística pela Universidade de São Paulo.

Conflito de interesses: inexistente
$50 \%$ dos nascidos prematuros e de baixo peso sobreviviam, por causa dos cuidados da equipe com o controle da temperatura, prevenção das infecções e adequado aporte nutricional ${ }^{2,3}$. Porém há muita preocupação quanto a qualidade de vida que estes bebês terão no decorrer de seu desenvolvimento ${ }^{4,7}$. A equipe envolvida nos cuidados com o RNPT deve dar suporte para favorecer o bom desenvolvimento global da criança.

A fonoaudiologia atua nos aspectos relacionados à alimentação, ao contato mãe/bebê, ao desenvolvimento da linguagem e da audição ${ }^{4}$.

Para que a alimentação do recém-nascido (RN) seja eficiente, é necessário adequado desenvolvimento motor oral e função de sucção forte, rítmica e coordenada, além do reflexo de procura e sucção, vedamento labial completo, movimentos adequados de língua e mandíbula, ritmo de sucção, eclosões de sucção alternadas com pausas e coordenação entre sucção/deglutição/respiração ${ }^{8}$. Além destes fatores, deve-se levar em conta o peso corpóreo, 
a força muscular, o suporte de peso, a atenção, o contexto específico do ambiente e a complexidade da tarefa oferecida ${ }^{5}$.

O RNPT apresenta imaturidade global e do sistema sensório motor oral, impossibilitando a alimentação por via oral. Desse modo, os RNs abaixo de 34 semanas de idade gestacional são, geralmente, alimentados por sondas gástricas ${ }^{8,13}$.

Segundo a literatura os reflexos orais de procura e de deglutição surgem entre a $9^{a}$ e a $11^{\text {a }}$ semanas de vida fetal e o reflexo de sucção entre a $18^{\mathrm{a}}$ e $24^{\mathrm{a}}$ semanas. Já a coordenação entre a sucção, deglutição e respiração acontecem na $34^{\text {a }}$ semanas de gestação, porém só estará plenamente estabelecida na $37^{\text {a }}$ - semana ${ }^{8,9,14,15}$

Para o início da alimentação por via oral do RNPT deve-se levar em consideração alguns fatores importantes, como: idade gestacional corrigida, peso atual, saturação de oxigênio, frequência respiratória e freqüência cardíaca durante a alimentação, grau de estabilidade clínica, processo de maturação e desempenho da sucção não-nutritiva (SNN) ${ }^{12}$. Deve-se considerar ainda, a função termorreguladora e o sistema gastrointestinal, relacionado à imaturidade enzimática e funcional do estômago e intestino ${ }^{16}$.

O longo período de internação, o uso prolongado de sonda gástrica e a privação de estímulos sensoriais na região oral, dificultam a maturação oromotora do $\mathrm{RN}$ e a transição da dieta para via oral, retardando, assim, a alta hospitalar 4,12,13,16.

Nesse momento, é necessária a intervenção de um fonoaudiólogo com terapia específica para auxiliar na maturação do sistema sensório motor oral $4,13,17$.

Desta maneira, o uso da técnica de SNN e de estimulação oromotora diminuem os transtornos causados pelo uso prolongado da sonda gástrica, estimulam os movimentos envolvidos na sucção e a coordenação sucção/deglutição/ respiração $4,6,8,10,18,19$.

A técnica de SNN consiste na introdução do dedo mínimo enluvado na boca do RN, tocando na parte anterior do palato (papila palatina), onde desencadeia o reflexo de sucção. Este procedimento geralmente é realizado antes ou concomitantemente ao recebimento da dieta por sonda. Esta técnica permite que o RN evolua gradativamente diminuindo a instabilidade, adquirindo maior precisão dos movimentos de língua e mandíbula, maior coordenação entre sucção, respiração e padrão postural, deixando o bebê alerta por mais tempo. Além disso, a SNN propicia a maturação do reflexo de sucção, estimula o trânsito intestinal e permite ao RN associar a sucção à plenitude gástrica ${ }^{8,14}$, refletindo, assim, em sua maturação e organização neurocomportamental ${ }^{19,21}$.
A técnica de estimulação oromotora consiste em toques lentos e profundos nas bochechas, lábios, gengiva e língua, por aproximadamente um minuto em cada estrutura, sendo finalizada com a SNN. Esta técnica propicia movimentos passivos de língua e deglutições sucessivas, que além de fortalecer a musculatura oral auxilia na estabilidade e coordenação da sucção/deglutição/ respiração $18,19,22$

Em neonatologia, essas técnicas são utilizadas separadamente ou em conjunto, uma complementando a outra. Existem diversos trabalhos relatando a eficiência das técnicas, porém há poucos estudos comparando-as.

O objetivo do presente estudo foi verificar a influencia da estimulação oromotora e da SNN na prontidão para alimentação oral e na eficiência da alimentação por via oral em gemelares.

\section{APRESENTAÇÃO DO CASO}

Participaram do programa de intervenção dois RNPTs, gemelares, um do sexo masculino e o outro do sexo feminino, com idade gestacional corrigida de 35 semanas e 2/7 dias.

Os sujeitos foram selecionados dentre os RNPTs atendidos na Unidade Neonatal do Hospital Maternidade Leonor Mendes de Barros, em São Paulo (SP), no período de novembro de 2008 a janeiro de 2009. Os gemelares foram selecionados seguindo critérios de estabilidade clínica e semelhanças nas hipóteses diagnósticas.

Os dois RNs foram avaliados e reavaliados pela fonoaudióloga pesquisadora (DFBC), seguindo os itens do Instrumento de Avaliação da Prontidão do Prematuro para Início da Alimentação Oral ${ }^{23}$. Após a avaliação foi sorteada qual das técnicas cada $\mathrm{RN}$ receberia, independentemente do resultado da avaliação.

O RN que passou a ser chamado de RN1 recebeu a técnica de estimulação oromotora e o RN que passou a ser chamado de RN2 recebeu a técnica de SNN. Ambas as técnicas terapêuticas foram realizadas pela fonoaudióloga pesquisadora (DFBC), uma vez ao dia, durante dez dias, a partir da avaliação, com intervalo de dois dias, sábado e domingo. No $11^{\circ}$ dia de atendimento, os RNs foram reavaliados.

Este estudo foi previamente aprovado pelo Comitê de Ética em Pesquisa do Hospital Maternidade Leonor Mendes de Barros - Protocolo CEP 036/08, conforme a Resolução 196/96 do Conselho Nacional de Saúde. Os responsáveis pelos RNs receberam informações sobre a pesquisa e assinaram o Termo de Consentimento Livre e Esclarecido. 


\section{A) Avaliação Fonoaudiológica}

A avaliação da prontidão do $R N$ para alimentação foi realizada por meio do Instrumento de Avaliação da Prontidão do Prematuro para Início da Alimentação Oral ${ }^{23}$, o qual consta dos seguintes itens: idade corrigida, estado de organização comportamental, postura oral, reflexos orais e SNN. Cada um destes itens recebeu um escore de 0 a 2 e o escore máximo atingido foi de 36 pontos.

A eficiência da alimentação por via oral foi avaliada por meio da identificação do volume aceito por via oral em relação ao volume total prescrito e pela relação entre o volume aceito e o tempo de aceitação da dieta por via oral, os quais foram verificados pelas técnicas do copinho e da sonda-dedo (feeding-finger) ou translactação digital.

A técnica do copinho consiste em oferecer a dieta ao RN num copo de plástico milimetrado e esterilizado, na qual o RN sorve o leite do copinho por meio do canolamento da língua e de seu movimento ântero-posterior. Esta técnica avalia a maturação do reflexo de deglutição e a coordenação deglutição/respiração. A técnica da sonda-dedo consiste na oferta da dieta ao RN por meio de uma sonda gástrica curta (número 6) acoplada ao dedo mínimo do examinador, na qual o RN suga o dedo do examinador extraindo o leite. Esta técnica avalia a maturação dos reflexos de sucção e deglutição e a coordenação sucção/deglutição/respiração.

\section{B) Técnicas de estimulação}

O RN1 foi submetido a 10 sessões de terapia fonoaudiológica baseada no programa de estimulação oral ${ }^{21}$, que consiste em movimentos circulares na região das bochechas, deslizamento digital posteroanterior na região vestibular dos lábios e gengiva (primeiro de um lado e depois do outro, na parte superior e na parte inferior), deslizamento digital posteroanterior nas laterais da língua, com a duração de um minuto para cada movimento e finalizando com dois minutos de SNN, antes da oferta da dieta.

O RN2 foi submetido a 10 sessões de terapia fonoaudiológica com SNN, que consiste na sucção digital do dedo mínimo do pesquisador por dois minutos, antes da oferta da dieta.

\section{C) Reavaliação}

Após a realização das 10 sessões de terapia fonoaudiológica, a $11^{\text {a }}$ sessão foi utilizada para a reavaliação fonoaudiológica seguindo os mesmos itens da avaliação inicial.

Os resultados serão apresentados de forma descritiva, baseados nas pontuações obtidas pelos sujeitos na avaliação e reavaliação.

\section{RESULTADOS}

A Tabela 1 apresenta a caracterização de RN1 e de RN2 quanto aos itens: peso ao nascimento, peso atual, dias de vida, idade gestacional, idade gestacional pós-conceptual e histórico de oxigenoterapia. Por serem gemelares diferem apenas nos itens peso ao nascimento, peso atual e histórico de oxigenoterapia. Observa-se que essas diferenças são pequenas e não influenciaram na intervenção fonoaudiológica.

A Tabela 2 apresenta os valores dos escores obtidos por RN1 e RN2 na avaliação e na reavaliação da prontidão para alimentação por via oral. Observa-se aumento dos escores da avaliação para a reavaliação, atingindo o escore máximo. Nota-se que a pontuação foi semelhante em todos os itens, inclusive no valor total dos escores. Observa-se que ambas as técnicas tiveram resultados positivos nos RNs, elevando em 10 pontos o escore total.

Tabela 1 - Caracterização dos RN1 e RN2

\begin{tabular}{ccc}
\hline & RN1 & RN2 \\
\hline Peso ao nascimento & $1420 \mathrm{~g}$ & $1370 \mathrm{~g}$ \\
Peso atual (na avaliação) & $1500 \mathrm{~g}$ & $1320 \mathrm{~g}$ \\
Dias de vida & $19 \mathrm{~d}$ & $19 \mathrm{~d}$ \\
Idade gestacional & $326 / 7 \mathrm{~s}$ & $326 / 7 \mathrm{~s}$ \\
Idade gestacional pós conceptual & $352 / 7 \mathrm{~s}$ & $352 / 7 \mathrm{~s}$ \\
\hline Ventilação mecânica prolongada & 0 & 0 \\
CPAP & 0 & 0 \\
Funil de O2 & $8 \mathrm{~d}$ & $3 \mathrm{~d}$ \\
Cateter nasal & 0 & 0 \\
\hline
\end{tabular}

Legenda: $d$ = dias, $s$ = semanas. 
Tabela 2 - Comparação entre a avaliação e reavaliação dos RN1 e RN2 em relação aos itens do protocolo de prontidão do prematuro para início da alimentação via oral

\begin{tabular}{ccccc}
\hline & \multicolumn{2}{c}{ Avaliação } & \multicolumn{2}{c}{ Reavaliação } \\
\cline { 2 - 5 } & RN1 & RN2 & RN1 & RN2 \\
\hline Idade corrigida & 2 & 2 & 2 & 2 \\
Estado de consciência & 1 & 1 & 2 & 2 \\
Postura global & 2 & 2 & 2 & 2 \\
Tônus global & 2 & 2 & 2 & 2 \\
Lábios & 2 & 2 & 2 & 2 \\
Língua & 2 & 2 & 2 & 2 \\
Procura & 1 & 1 & 2 & 2 \\
Sucção & 2 & 2 & 2 & 2 \\
Mordida & 2 & 2 & 2 & 2 \\
Vômito & 0 & 2 & 2 & 2 \\
Movimento de língua & 2 & 2 & 2 & 2 \\
Canolamento de língua & 0 & 0 & 2 & 2 \\
Movimento de mandíbula & 2 & 2 & 2 & 2 \\
Força de sucção & 1 & 1 & 2 & 2 \\
Sucções por pausa & 0 & 0 & 2 & 2 \\
Manutenção do ritmo s/p & 1 & 2 & 2 & 3 \\
Manutenção do estado de alerta & 2 & 2 & 2 & 36 \\
Sinais de estresse & 2 & 2 & 36 & 2
\end{tabular}

Legenda: $s / p=$ sucção por pausa.

A Tabela 3 apresenta os dados referentes à eficiência da alimentação dos RNs 1 e 2. Observa-se aumento do aproveitamento da dieta, da avaliação para a reavaliação, com a utilização de ambas as técnicas.
A Tabela 4 apresenta o tempo, em dias, que os RNs permaneceram em uso de sonda gástrica após início da intervenção fonoaudiológica. Nota-se que os RNs permaneceram o mesmo tempo em uso de sonda gástrica, não havendo diferença com o tipo de técnica utilizada.

Tabela 3 - Comparação entre a avaliação e a reavaliação dos RN1 e RN2 em relação à eficiência da alimentação

\begin{tabular}{ccccc}
\hline & \multicolumn{2}{c}{ Avaliação } & \multicolumn{2}{c}{ Reavaliação } \\
\cline { 2 - 5 } & $\mathbf{R N ~ 1}$ & $\mathbf{R N 2}$ & $\mathbf{R N 1}$ & $\mathbf{R N 2}$ \\
\hline Volume prescrito & $30 \mathrm{ml}$ & $25 \mathrm{ml}$ & $30 \mathrm{ml}$ & $20 \mathrm{ml}$ \\
Aproveitamento da dieta: translactação digital & $66,6 \%$ & $64 \%$ & $100 \%$ & $100 \%$ \\
Aproveitamento da dieta: copinho & $0 \%$ & $60 \%$ & $100 \%$ & $100 \%$ \\
Tempo de aceitação da dieta & $5 \mathrm{~min}$ & $5 \mathrm{~min}$ & $3 \mathrm{~min}$ & $5 \mathrm{~min}$ \\
Relação volume/tempo & $4 \mathrm{ml} / \mathrm{min}$ & $0,4 \mathrm{ml} / \mathrm{min}$ & $10 \mathrm{ml} / \mathrm{min}$ & $4 \mathrm{ml} / \mathrm{min}$ \\
\hline
\end{tabular}

Tabela 4 - Comparação entre os RN1 e RN2 em relação ao tempo (em dias) de permanência com a sonda gástrica após início da intervenção fonoaudiológica

\begin{tabular}{cll}
\hline & RN1 & RN2 \\
\hline Uso de sonda gástrica & $14 \mathrm{~d}$ & $14 \mathrm{~d}$ \\
\hline
\end{tabular}




\section{DISCUSSÃO}

O estudo teve como objetivo verificar a influencia das técnicas de estimulação oromotora e SNN na prontidão para alimentação oral e na eficiência da alimentação oral, por isso, optou-se na escolha de RNs que, além de se enquadrarem nos critérios de inclusão, tivessem características semelhantes, tanto em sua caracterização, com relação a idade gestacional, idade gestacional pós-conceptual e dias de vida, quanto na pontuação obtida na avaliação da prontidão do prematuro para início da alimentação via oral.

Os RNs da pesquisa apresentaram maior dificuldade nos itens canolamento de língua, força de sucção e sucções por pausa, o que interferem nos estados de organização dos mesmos, dificultam seus desempenhos nas habilidades oromotoras e refletem na manutenção do ritmo de sucção ${ }^{17}$.

Pode-se observar que a intervenção fonoaudiológica teve êxito independentemente da técnica utilizada, visto a melhora de ambos RNs da avaliação para a reavaliação. Ambas as técnicas propiciaram melhora na prontidão para a alimentação oral e na eficiência da alimentação oral desses $\mathrm{RNs}^{7,8,10,12,19}$.

A estimulação da SNN acelera a maturação da sucção, melhorando o padrão e o desempenho na alimentação por via oral ${ }^{8}$, apesar de não se saber ao certo quais características da sucção são beneficiadas, e a estimulação oromotora auxilia na maturação/coordenação da musculatura envolvida na sucção melhorando sua eficiência ${ }^{21}$.

Outro estudo menciona que as características da SNN como canolamento de língua, movimento de língua e força de sucção melhoram com o aumento da idade corrigida ${ }^{8}$, além da experiência de receber algum estímulo por via oral ${ }^{24}$.

O uso de protocolos padronizados para avaliação e reavaliação, como utilizado nesta pesquisa, permite, através dos resultados, uma reflexão sobre planejamento terapêutico, condutas e sobre a eficácia da intervenção fonoaudiológica, a qual neste estudo observa-se eficaz para ambos os RNs.

No item eficiência da alimentação, no qual avaliou-se o aproveitamento da dieta, observa-se que números em percentuais permitem uma visão mais real do quanto o RN está aceitando do volume da dieta, em relação a um volume total prescrito pelo médico.

Com relação ao tempo de uso de sonda gástrica, ambos os RNs permaneceram em uso pelo mesmo tempo, o que demonstra que o tipo de técnica utilizada não teve influencia neste aspecto.

Tanto a técnica de estimulação oromotora quanto a técnica de SNN aumentam a capacidade do RN em se alimentar via oral por sucção em até 8,2 dias mais cedo do que $R N$ não estimulados ${ }^{19}$, pois essas técnicas organizam a sequência sucção/deglutição/ respiração durante a alimentação propiciando a maturação e coordenação destas funções ${ }^{25}$.

\section{CONCLUSÃO}

A intervenção fonoaudiológica com uso da estimulação oromotora e SNN promoveram melhora na prontidão para alimentação oral e na eficiência da alimentação oral nos RNs deste estudo.

\section{ABSTRACT}

Background: Preterm Newborns speech therapy with the use of oromotor stimulation and nonnutritive sucking. Procedures: two Twin Newborns took part in this speech therapy with corrected gestational age of 35 weeks and 2/7 days. The clinical speech assessment was performed in order to check the premature readiness for the beginning of oral feeding and the efficiency of oral feeding by some relations such as the accepted volume by the total prescribed volume and the accepted volume by the time of the diet acceptance. Later on we picked up the technique that each newborn would receive. The First newborn got the oromotor stimulation and the second newborn has got the nonnutritive sucking technique. They went 10 (ten) sessions each and have been revaluated. Results: after the stimulation procedures both newborns showed improvement in their oral reflexes and pattern of non-nutritive sucking and also improved the use of oral diet. Conclusion: both Newborns were benefitted from the speech therapy.

KEYWORDS: Infant, Newborn; Infant; Feeding; Deglutition; Deglutition Disorders; Techniques 


\section{REFERÊNCIAS}

1. Barradas J, Fonseca A, Guimarães CLN, Lima GMS. Relationship between positioning of premature infants in Kangaroo Mother Care and early neuromotor development. J Pediatr. 2006; 82:475-80.

2. Rugolo LMSS. Crescimento e desenvolvimento a longo prazo do prematuro extreme. J Pediatr. 2005; 81(1):S101-10.

3. Camelo JS, Martinez FE. Dilemas nutricionais no pré-termo extremo e repercussões na infância, adolescência e vida adulta. J Pediatr. 2005; 81(1 supl):33-42.

4. Tanaka CK, Messas CS. UTI neonatal: experiência fonoaudiológica em um hospital e pronto-socorro infantil. In: Andrade CRF. Fonoaudiologia em berçário normal e de risco. São Paulo: Lovise; 1996.p. 259-67.

5. Castro CG, Lima MC, Aquino RR, Eickmann SH. Desenvolvimento do sistema sensório motor oral e global em lactentes pré-termo. Pró-Fono. 2007; 19(1):29-38.

6. Fujinaga Cl, Zamberlan NE, Rodarte MDO, Scochi CGS. Confiabilidade do instrumento de avaliação da prontidão do prematuro para alimentação oral. Pró-Fono. 2007; 19(2):143-50.

7. Rodrigues J. Sucção nutritiva e não-nutritiva em recém-nascidos pré-termo: ritmo e taxa de sucção [monografia]. Santa Maria (RS): Universidade Federal de Santa Maria; 2007.

8. Neiva FCB, Leone CR. Sucção em recémnascidos pré-termo e estimulação da sucção. Pró-Fono. 2006; 18(2):141-50.

9. Araújo KCS. Estimulação sensoriomotora oral: aspectos práticos. In: Lopes SMB, Lopes JMA. Follow-up do recém-nascido de alto risco. Rio de Janeiro: Medsi; 1999. p.301-7.

10. Silva RNM. Efeitos da sucção não-nutritiva (SNN) no prematuro. In: Lopes SMB, Lopes JMA. Follow-up do recém-nascido de alto risco. Rio de Janeiro: Medsi; 1999. p.309-16.

11. Madureira DL. Deglutição em neonatos. In: Ferreira LP, Befi-Lopes DM, Limongi SCO. Tratado de fonoaudiologia. São Paulo: Roca; 2005. p.219-29.

http://dx.doi.org/10.1590/S1516-18462011005000015

RECEBIDO EM: 07/12/2009

ACEITO EM: 31/08/2010

Endereço para correspondência:

Diane Fernanda Bernal Calado

Rua Sebastião dos Santos, 118 - Guarulhos - SP

CEP: 07077-190

E-mail: diane.fono@gmail.com
12. Neiva FCB, Leone CR. Efeitos da estimulação sucção não-nutritiva na idade de início da alimentação via oral em recém-nascidos pré-termo. Rev Paul Pediatr. 2007; 25(2):129-34.

13. Neiva FCB. Sucção em recém-nascidos: algumas contribuições da fonoaudiologia. Pediatria. 2000; 22(3):264-70.

14. Xavier C. Assistência à alimentação de bebê hospitalizado. In: Barreto MCA, Brock R, Wajnstein R. Neonatologia: um convite à atuação fonoaudiológica. São Paulo: Lovise; 1998. p.255-76. 15. Mizuno K, Ueda A. The maturation and coordination of sucking, swallowing, and respiration in preterm infants. J Pediatr. 2003:36-40.

16. Caetano LC, Fujinaga Cl, Scochi GS. Sucção não-nutritiva em bebês prematuros: estudo bibliográfico. Rev Latino-Am Enferm. 2003; 11(2):232-6.

17. Xavier $C$. Intervenção fonoaudiológica em bebês de risco. In: Ferreira LP, Befi-Lopes DM, Limongi SCO. Tratado de fonoaudiologia. São Paulo: Roca; 2005. p.415-38.

18. Fucile S, GiseL E, Lau C. Oral stimulation accelerates the transition from tube to oral feeding in preterm infantis. J Pediatr. 2002; 141(2):230-6.

19. Pimenta HP, Moreira MEL, Rocha AD, Gomes Junior SC, Pinto LW, Lucena SL. Efeitos da sucção não-nutritiva e da estimulação oral nas taxas de amamentação em recém-nascidos pré-termo de muito baixo peso ao nascer: um ensaio clínico randomizado. J Pediatr. 2008; 84(5):423-7.

20. Pinelli J, Symington A. Non-nutritive sucking for promoting physiologic stability and nutrition in preterm infants. Cochrane Database Syst Rev. 2005; 19:CD001071.

21. Fucile S, Gisel EG, Lau C. Effect of an oral stimulation program on sucking skill maturation of preterm infants. Dev Med Child Neurol. 2005; 47:158-62.

22. Pickler RH, Frankel HB, Walsh KM, Thompson NM. Effects of nonnutritive sucking on behavioral organization and feeding performance in preterm infants. Nurs Res. 1996; 45:132-5.

23. Fujinaga $\mathrm{Cl}$. Prontidão do prematuro para início da alimentação oral: confiabilidade e validação clínica de um instrumento de avaliação [tese]. Ribeirão Preto (SP): Universidade de São Paulo; 2005.

24. Simpson C, Schanler RJ, Lau C. Early introduction of oral feeding in preterm infants. Pediatrics. 2002; 110(3):517-22.

25. Boiron M, Nobrega LD, Heront A, Saliba E. Effects of oral stimulation and oral support on non-nutritive sucking and feeding performance in preterm infants. Dev Med Child Neurol. 2007; 49:439-44. 